\title{
Corporate Social Responsibility in der wertorientierten Unternehmensführung
}

\author{
Corporate Social Responsibility wird aus be- \\ triebswirtschaftlicher Sicht kontrovers bewer- \\ tet. Doch findet eine Verknüpfung mit dem \\ Shareholder-Value-Ansatz statt, kann das CSR- \\ Konzept als Geschäftsstrategie aufgefasst \\ werden, die auch monetäre Gewinne bringt. \\ Von Henry Schäfer
}

D ie wissenschaftliche und unternehmenspraktische Auseinandersetzung mit dem Einfluss von Corporate Social Responsibility (im Folgenden CSR genannt) auf den wirtschaftlichen, das heißt finanziellen Erfolg eines Unternehmens, ist bereits seit den 1960er Jahren im Gange.

\section{CSR im Business Case - pessimistischer Standpunkt}

Seit dieser Zeit halten sich auch im Wesentlichen die zwei Einwände gegen CSR in der Unternehmenspolitik.

Zum einen ist das Management nicht in der Lage und auch gar nicht befugt zu bestimmen, welches die soziale Verantwortung des von ihm geführten Unternehmens ist. Das Unternehmen wird hier rein als Einkommensquelle für die Eigenkapitalgeber angesehen. Es gilt, für sie den höchstmöglichen Wertbeitrag beziehungsweise die Ausschüttung pro Periode bereitzustellen. Den Eigenkapitalgebern obliegt es dann zu entscheiden, für welche Zwecke sie ihre Ausschüttungen und realisierten Kursgewinne verwenden wollen. Zum Beispiel zum eigennützigen Konsum beziehungsweise Sparen oder zu gemeinnützigen Ausgabenverwendungen wie Spenden. Die Verantwortung des Managements erschöpft sich nach dieser Sichtweise darauf, die Einkommensquelle der Eigenkapitageber höchstmöglich profitabel zu machen. „The only responsibility of a corporation is to deliver a profit to its shareholders." (1)

Zum anderen wird insbesondere darauf abgestellt, dass CSRMaßnahmen in Unternehmen in der Regel kurzfristig zu finanziellen Belastungen führen. Solche Ausgaben können erst in den Folgejahren, daher eher mittel- bis langfristig, und zudem sehr unsicher wieder verdient werden. Unter diesen Umständen gibt es keine finanziellen Anreize für die Eigenkapitalgeber und das Management, eine Unternehmenspolitik an den Grundsätzen der CSR auszurichten.
Zusammengefasst begründen sich die ablehnenden Haltungen der Gegner von CSR aus der Befürchtung, tendenziell keine Nettogewinne, sondern eher Nettoverluste zu realisieren und dadurch das Vermögen der Eigenkapitalgeber zu schmälern. Diese Befürchtung fällt umso mehr ins Gewicht, je ausgeprägter eine Kurzfristorientierung der Eigenkapitalgeber ist. Unter solchen Umständen dürften viele CSR-Maßnahmen mit ihrem hohen Grad an mittel- bis langfristiger Reifedauer und ihren inhärenten Unsicherheiten kaum wirtschaftlich sein.

\section{CSR im Business Case - optimistischer Standpunkt}

CSR-Maßnahmen sind in dieser Konzeption eingebettet in den Kontext von Managementtheorien und -praktiken. Es werden Begründungen dafür geliefert, dass CSR-Maßnahmen in den Wertschöpfungsprozessen von Unternehmen integriert zu verstehen sind. Die geschäftspolitischen CSR-Maßnahmen müssten in diesem Sinn die Kernkompetenzen eines Unternehmens stärken. Sie müssten dessen Dynamic Capabilities erhöhen und dadurch den Unternehmenswert tendenziell steigen lassen. CSR-Maßnahmen in Unternehmen stellen in weiten Teilen vor allem Wertbeiträge zum intangiblen Vermögen, wie beispielsweise der Unternehmensreputation, dar. Zentral ist dabei die Erkenntnis, dass es nicht staatliche Auflagen im Umweltoder Sozialbereich sind, die den Wettbewerbsvorteil eines Einzelunternehmens begünstigen. Denn solche Auflagen sind für alle Unternehmen gleichermaßen gültig und lassen ein zwischen Unternehmen sehr ähnliches reaktives Verhalten folgen. Erst die Erkenntnis und Akzeptanz in der Unternehmensleitung, dass Umwelt- und Sozialpolitik unternehmensindividuell, unternehmensstrategisch und proaktiv zu verstehen ist, kann Veränderungen in den Kernkompetenzen und Dynamic Capabilities zwischen einzelnen Unternehmen begründen und dadurch Wettbewerbsvorteile generieren. So weisen zahlreiche wissenschaftliche Forschungsarbeiten mittlerweile nach, dass erst eine Integration von CSR in die Unternehmensstrategie und deren unternehmenspolitische Umsetzung vor allem durch Anpassungen, Neuausrichtungen und Veränderungen im Wertschöpfungsprozess einen herausgehobenen Beitrag zum Unternehmenserfolg erbringen können (2).

Im Einzelnen werden in diesem Verständnis bestimmte Antriebskräfte von CSR auf den Unternehmenswert gesehen, die in Abbildung 1 im Überblick dargestellt sind und im Folgenden erläutert werden. 
Abbildung 1: Übersicht zu den Haupttreibern von CSR und ihren Effekten

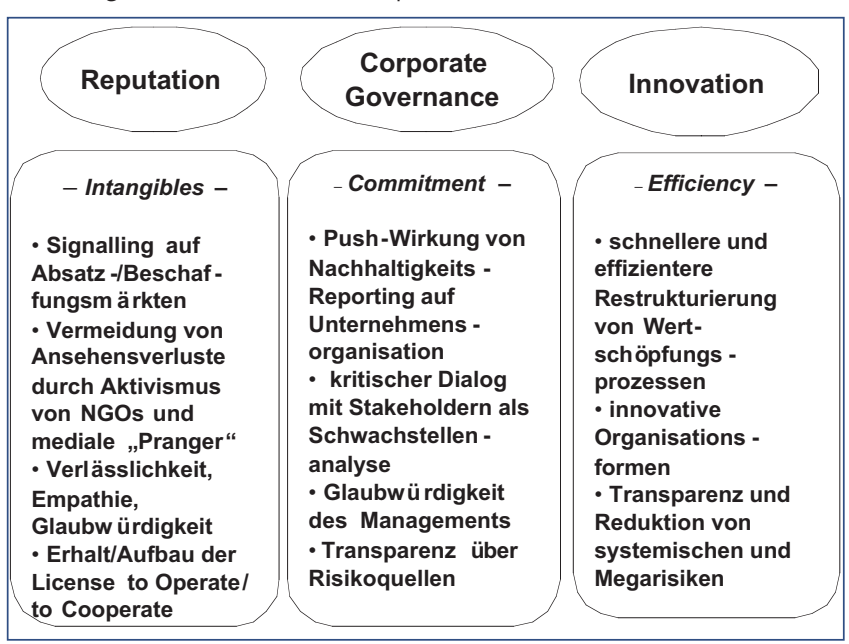

Quelle: Schäfer/Lindenmayer 2005

\section{Reputation}

CSR-Maßnahmen können dem Aufbau von Reputation gegenüber Stakeholdern dienen, indem sie Zuverlässigkeit, Glaubwürdigkeit, Fairness, Empathie und andere vertrauensbildende Eigenschaften signalisieren. Sie sind tendenziell dazu geeignet, dass Stakeholder ihre Informationsdefizite und Skepsis hinsichtlich CSR eines Unternehmens abbauen und eine mittel- bis längerfristige Beziehung eingehen (3).

Besonders deutlich wurden solche Effekte im Bereich der Human Resources erkannt. So konnte für Unternehmen, die besondere Investitionen in ihr Human-Resource-Management tätigen, nachgewiesen werden, dass sie in besonderem Maße hoch qualifizierte Arbeitskräfte auf sich lenken können (4). Neben der Stakeholdergruppe der Beschäftigten sind Steigerungen des Unternehmenswerts durch reputationsbildende Maßnahmen mittels CSR auch für Kunden von Unternehmen nachgewiesen. Dies gilt insbesondere für Kunden, die Wert auf derartige Maßnahmen legen (5). Ähnliches gilt im übertragenen Sinne auch für Lieferanten. Von besonderer Bedeutung können ebenso Kapitalgeber sein, etwa wenn sie als ethische oder nachhaltige Geldanleger in Aktien beziehungsweise Anleihen solcher Untenehmen bevorzugt anlegen, denen eine herausragende CSR attestiert wurde (6).

Reputationseffekte werden aber auch durch Unternehmensmaßnahmen im Bereich des Corporate Citizenship und auf philanthropischen Gebieten geschaffen. Reputationsbildung erhält oder erhöht damit die „License to Operate“ und „License to Cooperate“ eines Unternehmens innerhalb seines gesellschaftlichen Umfelds und stabilisiert so das Stakeholderumfeld für Unternehmensentscheidungen.

\section{Corporate Governance}

Ein nach Grundsätzen oder mit Elementen der CSR geführtes Unternehmen kann als Signal für eine besonders hohe Qua- lität und Kompetenz der Unternehmensleitung verstanden werden. In theoretischen und empirischen Studien konnten derartige Beziehungen nachgewiesen werden (7). So impliziert etwa eine Ausrichtung der Unternehmenspolitik auf CSR eine Zukunfts- und Langfristorientierung des Managements (8).

Der offensive und proaktive Umgang mit sozialen und ökologischen Themen befähigt Unternehmensleitungen in besonderer Weise zum frühzeitigen Erkennen von systemischen Risiken und Megarisiken. Dies gilt auch für Mikro- und Makrotrends, die zu Wettbewerbsvorteilen führen können. Die Installation von kritischen Dialogen mit Stakeholdergruppen kann als eine Form der Schwachstellenanalyse verstanden werden. Nachhaltigkeits-Reportings wiederum erfordern spezielle Organisationsstrukturen, um die erforderliche Datenmenge und -güte zur Verfügung zu stellen. Sie fördern dadurch auch eine neue Dimension von Unternehmenstransparenz.

\section{Innovation}

Vor allem im Umweltbereich konnte in empirischen Studien nachgewiesen werden, dass Unternehmen mit hohen Umweltbelastungen oft auch Unternehmen mit ineffizienter Beschaffungs- und Produktionsweise sind (9). Dadurch büßten sie unter anderem auch ihre relative Wettbewerbsstärke ein und reduzierten somit den eigenen Unternehmenswert. So ist eines der Erkenntnisse des Öko-Effizienz-Konzepts in der Unternehmensführung, dass eine proaktive Umstellung von Beschaffungs- und Produktionsprozessen zu moderneren Produktionsweisen, effizienteren Organisationsformen und Entwicklungsvorsprüngen führt.

\section{CSR im Investment Case}

In einem monetär organisierten, marktwirtschaftlichen System werden aufgrund der Anspruchsgruppen die Finanzmärkte eine herausgehobene Rolle in einem solchen Transmissionsprozess spielen (10):

Im Bereich der Stakeholdergruppe der Kapitalgeber versetzt die Verfügbarkeit von Informationen zur CSR Eigenkapitalgeber in die Lage, die Bereitstellung von Finanzmitteln an Unternehmen vom Ausmaß der erreichten CSR abhängig zu machen. In der Praxis der so genannten nachhaltigen Geldanlage sind zu diesem Zweck mittlerweile ganz bestimmte Anlagestrategien im Einsatz. Einem Missverständnis sei hier jedoch vorgebeugt: Anleger, die CSR-Aspekte mit in ihrer Anlagepolitik berücksichtigen, müssen deshalb nicht zwangsläufig ethisch ausgerichtet sein. So wird nachfolgend im Wesentlichen auf konventionelle Anleger abgestellt, die nur dann in Aktien von Unternehmen mit ausgeprägter CSR-Politik investieren, wenn dies eine Aussicht auf überdurchschnittliche Rendite bei gleichem Risiko oder auf gleichbleibende Rendite bei geringerem Risiko bietet.

CSR-Ratings und -Reports ermöglichen aber auch Banken und vergleichbaren kreditgebenden Finanzinstitutionen eine Disposition ihrer Finanzmittel nach Umwelt- und Sozial- 
kriterien. So haben 1992 die ersten Bankenvertreter die „UNEPErklärung der Finanzinstitute zur Umwelt und zur nachhaltigen Entwicklung“ unterzeichnet (11). Damit wird anerkannt, „(...) dass ökologische und soziale Risiken gleichzeitig Kreditausfall-, Haftungs- und Reputationsrisiken sind“ (12). Mit der derzeit in Gang befindlichen Umsetzung der internationalen bankenaufsichtsrechtlichen Auflagen zur Kreditbeurteilung und -entscheidung mittels bankinterner Credit-Rating-Systeme werden vor allem weiche und kontrastarme Informationen Verwendung finden. Hier wird eine direkte Befruchtung von Credit Ratings der Banken durch CSR-Ratings möglich (13). Damit wird der Analysehorizont von Banken hinsichtlich der Kreditwürdigkeit eines Unternehmens und seines Managements nicht nur um neue Risikoverständnisse wie etwa der Bedeutung systemischer Risiken erweitert. Vor allem erlangt auch die Zukunftsfähigkeit des Managements und der Unternehmensstrategie eine höhere Bedeutung.

Eine weitere wichtige Rolle zur Steuerung von Unternehmensverhalten und -risiko vor dem Hintergrund einer Geldwirtschaft übernehmen bereits heute schon Versicherungsgesellschaften. Zum einen sind sie im Rahmen des treuhänderischen Managements von Versicherungsgeldern institutionellen Investoren gleichzustellen und damit Kapitalgeber von Unternehmen. Insofern treffen in dieser Rolle für Versicherungsgesellschaften die oben ausgeführten Aussagen für Kapitalgeber mit $\mathrm{zu}$. Eine herausgehobene Bedeutung vermögen Versicherungsgesellschaften aber in ihrem eigentlichen Geschäftszweck, dem Absichern von Risiken und der Vorsorge zur Vermeidung von Risiken zu spielen. Entsprechend ihrer ökonomischen Funktion sind Versicherungsgesellschaften bestrebt, den zugesagten Risikoschutz wirtschaftlich für sie tragfähig zu halten. Hieraus entsteht ein wirtschaftlicher Anreiz für Versicherer, nach Informationen zur frühzeitigen und möglichst umfassenden Erkennung spezifischer Risiken der Versicherungsnehmer zu suchen.

Die hohe globale Vernetzung von Unternehmen, zunehmendes Auftreten von Klimakatastrophen, hohe Schadenszahlungen aufgrund von Produkthaftungen sowie Schäden durch bislang ungekannte Ausmaße von moralischem Risiko durch Unternehmensleitungen machen deutlich, dass zunehmend Risiken mit großen wirtschaftlichen Folgen für Versicherer zahlreiche CSR-Aspekte von Unternehmen betreffen.

\section{Transmission von CSR aus dem Business Case in den Investment Case - die Rolle der Finanzmärkte}

Vor allem große Unternehmen konkurrieren heutzutage in einer monetären Welt um den Erhalt und die Erweiterung ihrer Eigen- und Fremdkapitalbasis auf globalisierten Finanzmärkten. Im Zuge dieser Konkurenzsituation werden solche Unternehmensstrategien und -politiken durch das Management ergriffen, die den höchstmöglichen wirtschaftlichen Erfolg im Sinne einer Mehrung des Shareholder-Values erzielen. Unternehmensmaßnahmen mit Bezug zu CSR müssen in dieser Denkhaltung ei- nen erkennbaren Wertbeitrag zum Shareholder Value erzielen. Über die globalen Finanzmärkte findet daher nicht ohne Grund eine zentrale Transmission von CSR als Business Case mit dem Investment Case statt. Abbildung 2 gibt hierzu eine strukturierte Vorstellung im Überblick.

Abbildung 2: Verbindung und Übertragungswege von CSR zwischen Business Case und Investment Case

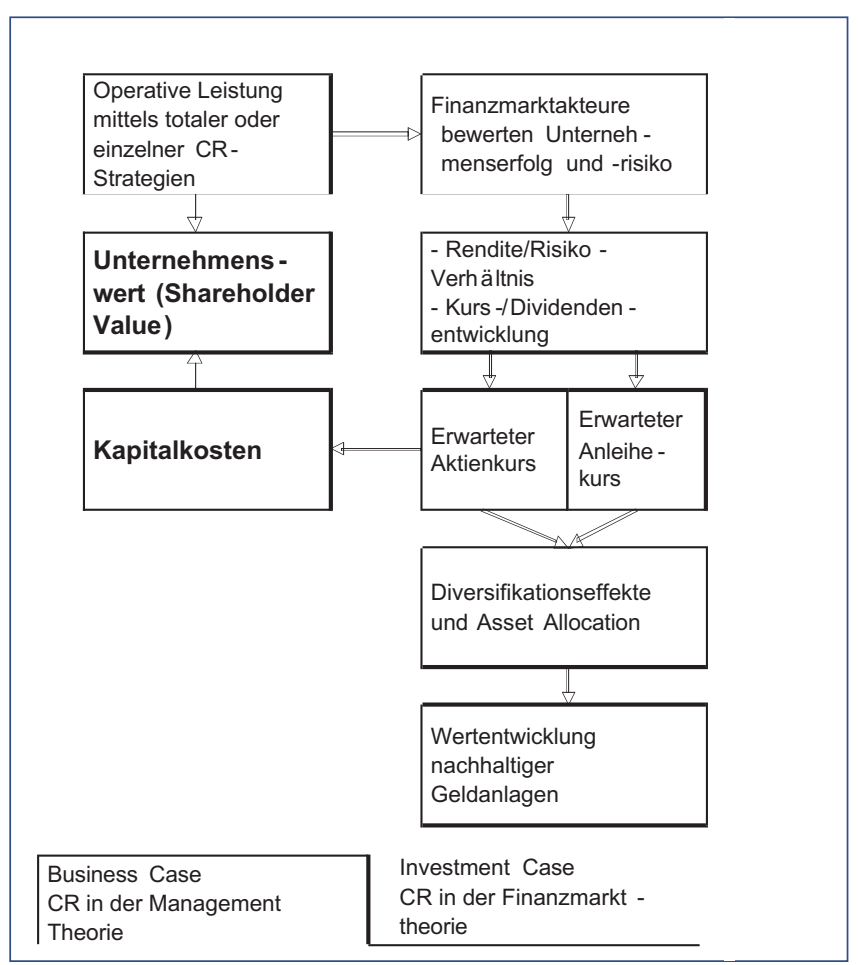

Quelle: Schäfer/Lindenmayer 2005

CSR kann innerhalb eines Unternehmens in erster Linie in den operativen Maßnahmen auf den Unternehmenserfolg wirksam werden. CSR-Maßnahmen der Unternehmensleitung stellen für die Beurteilung des zukünftigen Erfolgs und des Risikos eines Unternehmens wichtige Informationen für Finanzmarktakteure wie Rating-Institutionen, institutionelle Anleger, Kreditinstitute oder Versicherungsgesellschaften dar. Sie werden auf der Grundlage verfügbarer Informationen zur CSR eines Unternehmens ihre Erwartungen hinsichtlich der zukünftigen Kursentwicklung der Aktie und damit auch der Rendite/Risiko-Relation der Anlage in der Aktie des Unternehmens treffen. Dies berührt die geforderte Rendite der Kapitalgeber, zu denen sie bereit sind, dem betrachteten Unternehmen ihr Kapital weiterhin zu überlassen, beziehungsweise neu ihr Kapital in einem Unternehmen anzulegen.

So kann zum Beispiel die Erhöhung der Reputation des Unternehmens durch CSR-Maßnahmen in die Sicherheit der Produktion auf den Finanzmärkten als positiver Beitrag zur Reduktion des Risikos einer Kapitalanlage in die Aktie dieses Unternehmens verstanden werden. In Folge werden die Kapitalanleger nur noch eine geringere Kompensation für das ein- 
zugehende Risiko in der Anlage in der Aktie dieses Unternehmens verlangen. Daraufhin wird das betrachtete Unternehmen auch eine Reduktion seiner Kapitalkosten registrieren. Die Aufnahme von zusätzlichem Eigenkapital wird somit erleichtert und verbilligt. Reduktion von Unternehmensrisiken durch CSRMaßnahmen betrifft daher zwei Seiten der gleichen Medaille: bei den Kapitalgebern reduziert es den Anspruch auf die Höhe der Risikoprämie und bei der Kapital aufnehmenden Unternehmensleitung senkt es die Kapitalkosten.

Da der Shareholder-Value in der Finanzierungstheorie und -praxis als so genannter Gegenwartswert aller zukünftiger Gewinne eines Unternehmens zu verstehen ist, erhält der Kapitalkostensatz als Diskontierungsfaktor eine zentrale Rolle in der Bestimmung der Höhe des Shareholder-Values.

Kann der Kapitalkostensatz durch die beschriebene Reduktion des Risikos eines Unternehmens aufgrund der CSR-Maßnahmen gesenkt werden, so erhöht sich im Umkehrschluss der Wert des Unternehmens. Umgekehrt sinkt der Unternehmenswert, wenn der Kapitalkostensatz ansteigt - stets unter der Voraussetzung, dass sich in den übrigen Parametern, vor allem den Gewinnen und Ausschüttungen, nichts ändern würde. Nachfolgende Gleichung fasst diese Zusammenhänge in vereinfachter Form zusammen:

Shareholder Value $=\sum_{\text {Periode (Anfang) }}^{\text {Periode (Ende) }} \cdot \frac{\text { Gewinne der Zukunft }}{(1+\text { Kapitalkostensatz })^{\text {Periode }}}$

Die vorgenannten Aussagen treffen im Wesentlichen gleichermaßen für Aktien und Anleihen als Finanzierungsinstrumente der Unternehmensleitung zu. Aus Sicht der Kapitalgeber werden die so von ihnen erworbenen oder veräußerten Aktien oder Anleihen die individuellen Portfolios verändern. Dabei werden vor allem professionelle Anleger den Empfehlungen und Erkenntnissen der Portfolio-Theorie folgen und Kapitalanlagen insgesamt so zusammenstellen, dass sich die Risiken und Renditen der einzelnen Anlagetitel gegenseitig weitgehend neutralisieren (14). Es werden allerdings bei streng auf Rendite-Risiko-Beziehungen achtenden Anlegern immer nur solche Aktien von CSR-Unternehmen in die Portfolios aufgenommen, die zur Erhöhung des Gesamtwerts individueller Kapitalanlagen beitragen. Über diesen Sanktionsmechanismus werden letztendlich alle Unternehmensmaßnahmen gesteuert, auch die aus dem CSR-Bereich (15).

\section{Fazit und Ausblick}

Werden auf betriebswirtschaftlicher Ebene das Shareholder Value- und das CSR-Konzept im Rahmen einer ökonomisch wertorientierten Unternehmensführung miteinander verwoben, so lassen sich CSR-Strategien als Geschäftsstrategien verstehen, die darauf abzielen, sowohl den sozialen und/oder ökologischen Nutzen interner und externer Stakeholder als auch den monetären Wert für die Gruppe der Shareholder zu erhöhen. Quasi wird eine Win-Win-Situation angestrebt. Damit sind CSR-Strategien

\section{„CSR kann innerhalb eines Unternehmens in erster Linie in den operativen Maßnahmen auf den Unternehmenserfolg wirksam werden."}

rein ökonomisch begründbar. Die ökologischen und die sozialen Ziele können quasi als Metaziele aufgefasst werden, die dem ökonomischen Ziel der Maximierung des Shareholder Value dienen. Sie sind zudem integraler Bestandteil der Wertschöpfungsprozesse.

In einer solchen Denkhaltung steht die Identifikation von unternehmensspezifischen CSR-Strategien und -Maßnahmen zu Beginn im Vordergrund, deren Umsetzung faktisch wie Werttreiber wirken. Im Umkehrschluss gilt dann, dass CSRMaßnahmen ohne positiven Beitrag zum Unternehmenswert im Sinn des Shareholder-Value aus Sicht der Anteilseigner und des Managements überflüssigen Luxus darstellen, der aufgrund der ökonomischen Maßgabe nicht umgesetzt werden sollte.

An dieser Stelle tritt die Diskussion um die wirtschaftliche Einbettung von CSR in den Unternehmenskontext in Beziehung zu einer Diskussion, die Dynamic Capabilities und Kernkompetenezen als Handlungsmöglichkeiten von Unternehmensleitungen versteht, flexibel auf sich häufig ändernde Zustände im Handlungsumfeld des Unternehmens reagieren zu können. Forschungsarbeiten zeigen, dass zu diesem Zweck das Shareholder-Value-Konzept der wertorientierten Unternehmensführung durch optionale Wertbeiträge angereichert werden kann. CSR-Maßnahmen als Quellen zukünftiger Handlungsflexibilität zur Erhöhung der Wandlungsfähigkeit von Unternehmen, ganz im Sinne des Konzepts der nachhaltigen Entwicklung, dürften so auch für die nächste Generation von Bewertungs- und Steuerungsmodellen im CSR-Kontext eine hohe Bedeutung zukommen.

\section{Anmerkungen}

(1) Friedman, 1970

(2) Vgl. hierzu Hart/Ahuja, 1996.

(3) Vgl. Leisinger, 2003

(4) Vgl. hierzu die empirischen Ergebnisse von Turban/Greening, 1996.

(5) Vgl. z.B. Ginsberg/Bloom, 2004.

(6) Vgl. Schäfer, 2001 und Schäfer, 2003.

(7) Vgl. etwa Waddock/Graves, 1997.

(8) Vgl. Shrivastava, 1995.

(9) Vgl. hierzu etwa die Arbeit von Russo/Fouts, 1997.

(10) Vgl. Schäfer/Lindenmayer, 2005.
} 
(11) Vgl. UNEP, 1997.

(12) Kubusch, 2004, S. 10

(13) Vgl. Schäfer 2004

(14) Vgl. Schäfer, 2005, S. 277ff..

(15) Vgl. zum gesamten Abschnitt Schäfer/Lindenmayer, 2005

\section{Literatur}

Friedman, M.: The Social Responsibility of Business Is To Increase Its Profits. New York Times Magazine, 13.09.1970. S. 33.

Ginsberg, J.M. / Bloom, P.N.: Choosing the Right Green Marketing Strategy. MIT Sloan Management Review, Fall 2004. S. 79-84.

Hart, S.L. / Ahuja, G.: Does It Pay to Be Green? An Empirical Examination of the Relationship between Emission Reduction and Firm Performance. Business Strategy and the Environment, Vol. 5, 1996. S. 30-37.

Henderson, D.: Misguided Virtue: False Notion of Corporate Social Responsibility. Institute of Economic Affaires, London 2002.

Kubusch, J.: Aktuelle Entwicklungen bei der Erfassung von ökologischen und sozialen Risiken im Kreditvergabegeschäft. FORUM Wirtschaftsethik, 12. Jg., H. 2, 2004. S. 10-11.

Leisinger, K.M.: Whistleblowing und Corporate Reputation Management. München/Mering 2003.

Pleon Kothes Klewes GmbH: Geheime Mission? Deutsche Unternehmen im Dialog mit kritischen Stakeholdern. Eine Umfrage unter den 150 größten Unternehmen. Bonn, Berlin 2004.

Prahalad, C. K. /Hamel, G.: The Core Competence of the Corporation. Harvard Business Review, May-June, 1990. S. 79-91.

Russo, M.V. / Fouts, P.A.: A Resource-Based Perspective on Corporate Environment Performance and Profitability. Academy of Management Journal, Vol. 40 (4), 1997. S. 534-559.

Schäfer, H.: Unternehmensinvestitionen, Grundzüge in Theorie und Management, 2. Aufl. Stuttgart 2005.

Schäfer, H.: Unternehmensnachhaltigkeit und Ausfallrisiko im Kontext von Basel II. FORUM Wirtschaftsethik, 12. Jg., H. 2, 2004. S. 4-9.

Schäfer, H. (unter Mitarbeit von Preller, E.): Nachhaltigkeit von Unternehmen aus Finanzmarktsicht - Konzepte der Nachhaltigkeitsmessung auf Finanzmärkten des deutschsprachigen Raums, Edition der Hans Böckler Stiftung, Nr. 84. Düsseldorf 2003.

Schäfer, H.: Triple Bottom Line Investing - Ethik, Rendite und Risiko in der Kapitalanlage. Zeitschrift für das gesamte Kreditwesen, 54. Jg., H. 13, 2001. S. 740-744.
Schäfer, H. / Lindenmayer, Ph., Unternehmenserfolge erzielen und verantworten. Ein finanzmarktgesteuertes Beurteilungs- und Steuerungsmodell von Corporate Responsibility, Forschungsbericht, Bertelsmann-Stiftung, Gütersloh. 2005, download unter http://www.bertelsmann-stiftung.de/cps/rde/xbcr/SID-0A000F0AEF245CE8/bst/xcms_bst_dms_16297_16298_2.pdf, Zugriff: 105.07.2006.

Schäfer, H. / Stederoth, R.: Performance von Screened Portfolios - Stand der empirischen Ergebnisse in der Kapitalmarktforschung. Kredit und Kapital, 35. Jg., H. 1, 2002. S. 101-148.

Shrivastava, P.: Ecocentric Management for a Risky Society. Academy of Management Review, Vol. 20, 1995. S. 118-137.

Turban, D.B. / Greening, D.W.: Corporate Social Performance and Organizational Attractiveness to Prospective Employees. Academy of Management Journal, Vol. 40 (3), 1996. S. 658-672.

UNEP - United Nations Environment Programme: UNEP-Erklärung der Finanzinstitute zur Umwelt und zur nachhaltigen Entwicklung. Revidierte Fassung vom Mai 1997. URL: http://www.unepfi.org/signatories/statements/fi/german/, Zugriff: 07.09.2005.

Waddock, S. A. / Graves, S. B.: The Corporate Social Performance - Financial Performance Link. Strategic Management Journal, Vol. 18 (4), 1997. S. 303-319.

I AUTOR + KONTAKT

Dr. Henry Schäfer ist Professor an der Universität Stuttgart und Leiter der Abteilung III des Betriebswirtschaftlichen Institutes.

Universität Stuttgart, Betriebswirtschaftliches Institut, Abteilung Finanzwirtschaft, Kronenstr. 39, 70174 Stuttgart. E-Mail: h.schaefer@bwi.uni-stuttgart.de

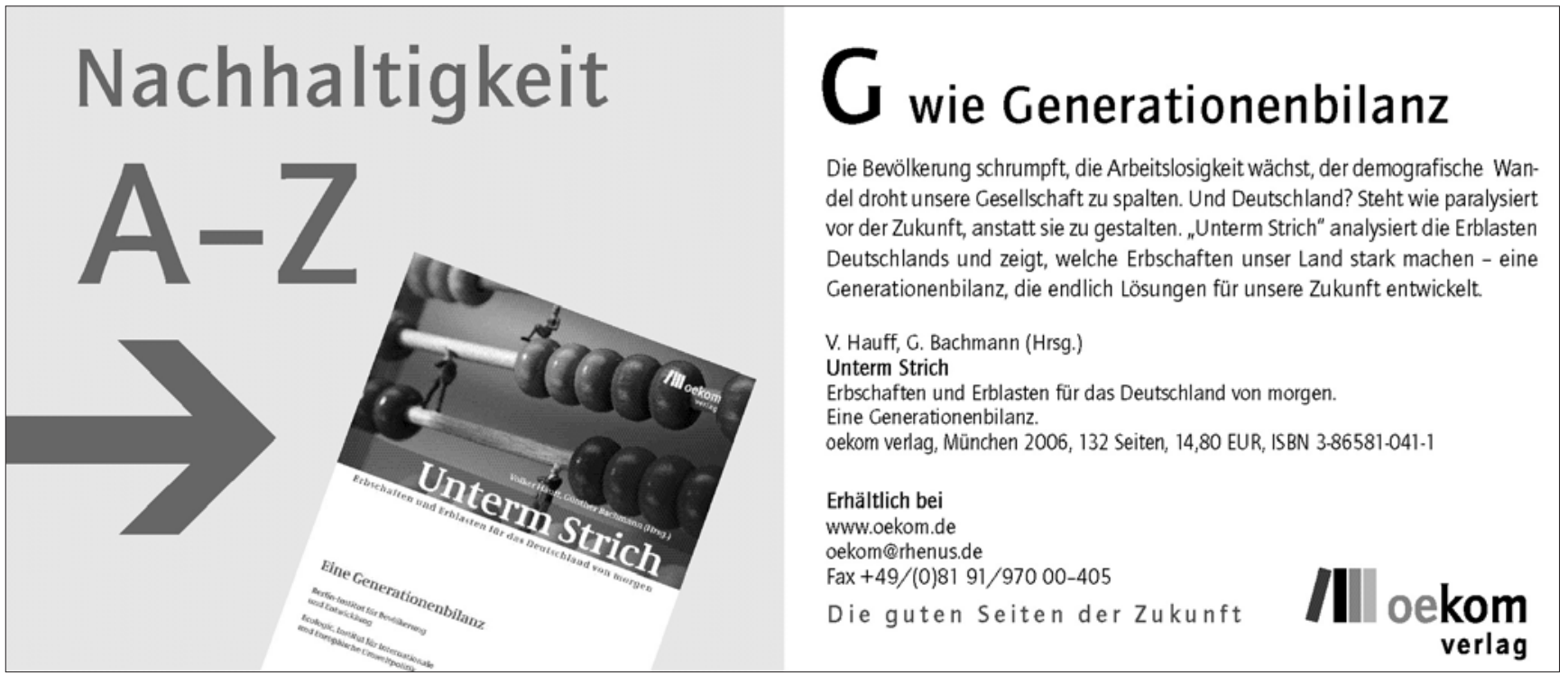


(c) 20I0 Authors; licensee IÖW and oekom verlag. This is an article distributed under the terms of the Creative Commons Attribution Non-Commercial No Derivates License (http://creativecommons.org/licenses/by-nc-nd/3.o/), which permits unrestricted use, distribution, and reproduction in any medium, provided the original work is properly cited. 